\title{
On the performance of adaptive coding schemes for energy efficient and reliable clustered wireless sensor networks
}

\author{
Imad Ez-zazi ${ }^{\mathrm{a}, *}$, Mounir Arioua ${ }^{\mathrm{a}}$, Ahmed El Oualkadi ${ }^{\mathrm{a}}$, Pascal Lorenz ${ }^{\mathrm{b}}$ \\ a Laboratory of Information and Communication Technologies (LabTIC), Abdelmalek Essaadi University, ENSA of Tangier, Morocco \\ ${ }^{\mathrm{b}}$ GRTC Laboratory, Network and Telecommunication Department, IUT-University of Haute Alsace, Colmar, France
}

\section{A R T I C L E I N F O}

\section{Article history:}

Received 31 January 2017

Revised 18 June 2017

Accepted 7 July 2017

Available online 8 July 2017

\section{Keywords:}

Wireless sensor networks

Clustering

Channel coding

Energy efficiency

Reliability

\begin{abstract}
A B S T R A C T
Clustering is the key for energy constrained wireless sensor networks (WSNs). Energy optimization and communication reliability are the most important consideration in designing efficient clustered WSN. In lossy environment, channel coding is mandatory to ensure reliable and efficient communication. This reliability is compromised by additional energy of coding and decoding in cluster heads. In this paper, we investigated the trade-off between reliability and energy efficiency and proposed adaptive FEC/FWD and FEC/ARQ coding frameworks for clustered WSNs. The proposed schemes consider channel condition and inter-node distance to decide the adequate channel coding usage. Simulation results show that both the proposed frameworks are energy efficient compared to ARQ schemes and FEC schemes, and suitable to prolong the clustered network lifespan as well as improve the reliability.
\end{abstract}

(C) 2017 Elsevier B.V. All rights reserved.

\section{Introduction}

Wireless sensor network (WSN) is one of the most promising technologies due to their unique characteristics, low cost, easy deployment and flexibility [1]. This emergent technology has attracted significant attention in recent years in many applications $[2,3]$. WSN is composed of numerous sensor nodes dispersed autonomously in specific area of interest to gather a physical parameter or monitor environmental conditions [4]. Nodes typically powered by small and limited batteries which replacement is very difficult and expensive in hostile environment. Thus, nodes are expected to be stand-alone and able to run for many months or even years without batteries replacement [5]. Therefore, reducing energy consumption in order to prolong network lifespan is the most crucial requirement and challenge consideration for WSN $[6,7]$.

Communication reliability is another crucial factor in low power WSN $[8,9]$. In highly lossy environment, the radio signal is often affected by noise, interferences, multipath fading and shadowing. These encountered undesirable impairments result in significant packet loss and delayed receiving. The increase of transmission energy results in successful and reliable transmission. However, the rise of transmission energy profoundly affects the sensor nodes energy and the network lifetime. Designing optimal WSN entails

\footnotetext{
* Corresponding author.

E-mail addresses: ezzazi@ieee.org, imad.ezzazi@gmail.com (I. Ez-zazi), m.arioua@ieee.org (M. Arioua), ahmed.eloualkadi@ieee.org (A. El Oualkadi), lorenz@ieee.org (P. Lorenz).
}

providing reliable communication with the minimum required energy consumption. Using error control coding (ECC) is an efficient strategy commonly used to lower the required transmission energy along with protecting the transmitted packets from errors and packet loss $[10,11]$. In low power application such as WSN, applying adaptive and low power ECC techniques is a primordial requirement [12]. Moreover, a WSN requires a powerful channel coding scheme when the transmission distance outweighs certain threshold level. Although advanced coding schemes provide higher coding gain, they require higher energy consumption with their complex decoding algorithms. If the extra energy consumption at the decoder exceeds the transmitted power savings due to the use of coding, then coding would not be energy-efficient compared to an uncoded system. Thus, a trade-off between reliability and energy depletion should be considered in order to optimize the error control schemes in WSN [13].

Network architecture and routing design are relevant factors which affect reliability and energy efficiency in WSN [14]. Clustered networks based on clustering routing are widely adopted in WSN to manage the energy efficiency [15-17]. In cluster-based architecture, two types of nodes coexist in the network and form clusters. Source nodes $(\mathrm{S})$ which sense, encode and transmit their data, and cluster heads $(\mathrm{CH})$ which receive and decode data in one hand, and then aggregate, encode and transmit the data to the base station (BS) in the other hand. Indeed, when a node is selected as a $\mathrm{CH}$, the computation and energy burden become completely important. In this case, using powerful coders and complex decoders intensely affects and worsens the $\mathrm{CH}$ energy and the net- 
work efficiency. In the last few years, a large literature has been emerged on clustered WSN in various applications. However, most of existing approaches have focused on the design of routing protocols, compression techniques and multiple access architectures [18-21]. These approaches commonly assume that the transmitted sensors readings are well received by collector nodes $(\mathrm{CH} / \mathrm{Sink})$ without packet loss during the transmission and without coding effect on the system.

In this paper, we investigate the energy efficiency of the coding and decoding processes in clustered WSN performance. The concept of adaptive error control coding (AECC) is proposed along with presenting a substantial coding frameworks in clustered sensor network. The proposed adaptive frameworks are proved to be energy efficient in clustered WSN allowing to efficient ECC to be used conveniently and effectively in the network.

The remainder of this paper is organized as follows. Section 2 discusses the related works. The sensor energy and channel propagation models for WSN are described in Section 3. Section 4 shows an overview of ECC schemes in WSN and some of paramount coding techniques used in this work. The mechanism of the proposed coding frameworks is presented and discussed in Section 5. In Section 6, the simulation results are analyzed and discussed. Section 7 concludes the paper.

\section{Related work}

Recently, there have been several works focused on ECC performance in WSNs. Automatic Repeat Request (ARQ) and Forward Error Correction (FEC) strategies have been mainly taken into consideration to encounter packet transmission errors and ensure communication reliability [22]. ARQ techniques have been investigated in WSN and proven energy consuming when the channel state is time varying with multipath and fading, such that the number of retransmissions and control acknowledgments increases [12]. In FEC approach, many coding schemes have been evaluated, including Bose-Chaudhuri-Hocquengham $(\mathrm{BCH})$ codes, Reed Solomon (RS) codes, convolutional codes, Turbo codes and Low Density Parity Check (LDPC) codes [23-26]. Most of the FEC schemes have proved to be effective in the case of end to end communications when the decoding is carried out at the base station. For instance, RS codes have been proven suitable and energy efficient for WSN applications with simple and reasonable coding and decoding complexity [27]. Results presented in [28] show that using RS codes with short codeword length and Binary-Phase-Shift-Keying (BPSK) modulation scheme in WSN saves about $50 \%$ of energy. In another work, LDPC codes have been proven to own better performance and several advantages over other block and convolutional codes [29]. They are the most performant coding schemes and competitors of Turbo codes in terms of performance and complexity. Experimental results in $[30,31]$ exhibit that LDPC codes are good candidates for WSN applications. Further works have focused on Hybrid ARQ (HARQ) with both ARQ and FEC approaches in sensor network [32].

The effect of the coding schemes directly depends on the network architecture, which leads researchers to take the network topology in consideration along with the coding approach [12]. In multi-hop architecture, each node has to use coding and decoding each time it receives neighbor packets. In this case, a strong FEC that requires complex decoders with large hardware and energy consumption turns out to be inadequate for this kind of network [24]. Moreover, using ARQ with retransmissions in each node will result in major interferences and intensely shorten the network lifetime. For this reason, authors of [30] proposed the use of short-length LDPC codes in WSN in order to mitigate the LDPC decoder effect and provide acceptable Bit Error Rate (BER). An Adaptive Iterative Decoding (AID) approach exploiting a threshold on the number of iterations of decoding algorithm for a certain BER is proposed in [33]. This approach with iterative LDPC decoding lowered the network energy consumption by $20-25 \%$. Although LDPC codes exhibit higher performance in terms of reliability, the extra power consumption of LDPC decoders limits the use of these codes in energy constrained intermediate nodes. This leads authors of $[34,35]$ to suggest the use of strong LDPC codes in single-hop sensor network where decoding is considered only at the BS in order to save energy and prolong the network lifetime. Moreover, several works have been interested in the optimization and use of adaptive approach with strong coding techniques in multi-hop network design [36]. For instance, a low power decode-and-forward approach for multi-hop WSN is proposed in [37], where a Viterbi decoding of convolutional codes is implemented in intermediate nodes while the iterative decoding algorithm of Turbo codes is applied at the base station. In our last work, we have presented an adaptive coding approach for multi-hop WSN, in which strong and soft FEC coding schemes are adaptively adopted according to the inter-node distance and channel condition [38]. Further architecture is clustered sensor network where special nodes are selected to relay other nodes packets. This architecture has been proven to be more energy efficient than multi-hop networks and adopted in many applications [39]. However, the effect of the coding and decoding schemes in this kind of network have been neglected in many works. Clustered network typically aims to balance the energy consumption among all the network nodes by periodically electing $\mathrm{CH}$ in order to save energy. Nevertheless, using coding and decoding along with aggregation and transmission in these special nodes may intensely increase the energy and defect the efficiency of clustered network.

In this paper, we investigate the error control approaches effect on clustered WSN, where $\mathrm{CH}$ nodes have a heavy burden of both processing and transmission energy. Based on the channel condition and inter-node distance, we propose efficient coding frameworks in which $\mathrm{CH}$ nodes decide the adequate error control approach to use, while an iterative belief propagation (BP) decoding is implemented at the base station to correct and retrieve all packets.

\section{Channel propagation and energy model}

Many radio propagation models known for wireless communications predict the signal-strength loss with distance. The free space and multipath propagation models are widely used for WSNs [40]. In the free space model, communications between the transmitter and receiver are clear line-of-sight in which reflecting surfaces and effect of the earth surface are solely absent. The multipath propagation is as result of different signal paths between the transmitter and receiver. These paths are the major detriment to signal propagation by giving rise to interference, distortion of the signal, loss of data and multipath fading.

For both free space and multipath propagation, the received power decreases as the distance between the transmitter and receiver increases [41]. For this work, both the free space model and multipath fading model were used, depending on the distance between the transmitter and receiver. If the inter-node distance (distance between transmitter and receiver) is less than a crossover distance $\left(d_{c r}\right)$, the Friss free space model is used ( $d^{2}$ attenuation). Otherwise $\left(d \geq d_{c r}\right)$, the two-ray ground propagation model is adopted ( $d^{4}$ attenuation). The crossover distance for Friss and two-ray-ground attenuation models is notably related to antenna technology and defined in [42] as:

$d_{c r}=\frac{4 \pi \sqrt{L} h_{r} h_{t}}{\lambda}$ 
Where $h_{r}$ and $h_{t}$ are the heights of the receiving and transmitting antennas above the ground respectively, $\lambda$ is the transmitted wavelength corresponding to the transmitting frequency and $L \geq 1$ is the system loss factor not related to propagation (we consider a lossless system, $L=1$ ).

The transmit power is attenuated according to the Friss free space equation for distance bellow $d_{c r}$ as follow:

$P_{r}(d)=\phi \frac{P_{t}}{d^{2}}, \quad d<d_{c r}$

Where $\phi=\frac{G_{t} G_{r} \lambda^{2}}{(4 \pi)^{2} L}, P_{r}(d)$ is the receive power for a transmitterreceiver separation of inter-node distance $d, P_{t}$ is the transmit power, $G_{t}$ and $G_{r}$ are the gains of transmitting and receiving antennas, respectively and $d$ is the distance between the transmitter and receiver.

This attenuation is only considered when the transmitter and receiver have direct line of sight communication, which occurs when the inter-node distance is short $\left(d<d_{c r}\right)$. For all distances greater than $d_{c r}$, the transmit power is attenuated according to the two-ray ground propagation equation as follows:

$P_{r}(d)=\varphi \frac{P_{t}}{d^{4}}, \quad d \geq d_{c r}$

where

$\varphi=\frac{G_{t} G_{r} h_{t}^{2} h_{r}^{2}}{L}$

\subsection{Sensor energy model}

The energy consumption of sensor nodes is assessed by a real and practical energy model where both the radio energy $E_{\text {radio }}$ and the computation energy $E_{\text {comp }}$ are the major energy components. The sensor node energy consumption per bit is calculated as the sum of $E_{\text {radio }}$ and $E_{\text {comp }}$. Based on the energy models outlined in $[38,42-44]$, the energy consumption required to communicate (transmit and receive) $k$-bits message at each round $r$ is expressed as:

$E_{\text {tot }}^{(r)}=E_{\text {radio }}^{(r)}(k)+E_{\text {comp }}^{(r)}(k)$

The radio energy $E_{\text {radio }}^{(r)}$ is composed of energy expended in transmission $E_{T x}^{(r)}$ and reception $E_{R x}^{(r)}$ :

$E_{\text {radio }}^{(r)}=E_{T x}^{(r)}(k)+E_{R x}^{(r)}(k)$

In sensor nodes, the transmitter dissipates energy to run the radio electronics $\left(E_{c k t}^{(r)}\right)$ and the amplifier $\left(E_{a m p}^{(r)}\right)$; however, the receiver dissipates energy to run mainly the radio electronics. Thus, the energy expended to transmit $k$-bits message over a distance $d$ is described as:

$E_{T x}^{(r)}=E_{c k t}^{(r)}(k)+E_{a m p}^{(r)}(k, d)$

Considering free space and multipath propagation, the Eq. (6) is formulated as:

$E_{T x}^{(r)}= \begin{cases}k\left(E_{c k t}^{(r)}+\epsilon_{f s p} d^{2}\right) & \text { if } d<d_{c r} \\ k\left(E_{c k t}^{(r)}+\epsilon_{t r p} d^{4}\right) & \text { if } d \geq d_{c r}\end{cases}$

When receiving data, the energy expended is expressed as:

$E_{R x}^{(r)}=E_{c k t}^{(r)} k$

The radio electronic energy is determined based on the circuit power depletion $P_{c k t}$ and the bit rate $R$ [44]:

$E_{c k t}^{(r)}=\frac{P_{c k t}^{(r)}}{R}$
Table 1

Circuitry parameters [45].

\begin{tabular}{ll}
\hline Parameters & Value \\
\hline$\beta_{F S}$ & $13.7 \mathrm{~mW}$ \\
$\beta_{L N A}$ & $0.55 \mathrm{~mW}$ \\
$\beta_{B P F}$ & $6.12 \mathrm{~mW}$ \\
$\beta_{I F A}$ & $0.2 \mathrm{~mW}$ \\
$\beta_{L P F}$ & $0.29 \mathrm{~mW}$ \\
$\beta_{A D C}$ & $4.1 \mathrm{~mW}$ \\
$\beta_{D A C}$ & $55 \mathrm{~mW}$ \\
\hline
\end{tabular}

Where

$P_{c k t}=\beta_{D A C}+2 \beta_{L P F}+2 \beta_{F S}+2 \beta_{B P F}+\beta_{L N A}+\beta_{I F A}+\beta_{A D C}$

$\beta_{D A C}, \beta_{A D C}, \beta_{L P F}, \beta_{F S}, \beta_{B P F}, \beta_{L N A}$ and $\beta_{I F A}$ are the power consumptions in: digital-to-analog converter (DAC), analog-to-digital converter (ADC), low-pass filter (LPF), frequency synthesizer (FS), band-pass filter (BPF), low-noise amplifier (LNA), and intermediatefrequency amplifier (IFA), respectively.

The circuit component power summarized in Table 1 are based on $0.18 \mu \mathrm{m}$ CMOS technology [45].

The parameters $\epsilon_{f s p}$ and $\epsilon_{t r p}$ are the Friss free space and two ray multipath amplifier respectively, which depend on the required received sensitivity and the receiver noise figure, as the transmit power has to be tuned so that the power at the receiver is above a certain threshold $P_{r}^{(t h r)}$. The minimum transmit power is determined based on this received power threshold. The transmit power is equal to the transmit energy per bit $E_{a m p}(1, d)$ multiplied by the bit rate $R$ :

$P_{t}=E_{\text {amp }}(1, d) R$

Substituting in the value of $E_{a m p}(1, d)$ gives:

$P_{t}= \begin{cases}\epsilon_{f s p} R d^{2} & \text { if } d<d_{c r} \\ \epsilon_{t r p} R d^{4} & \text { if } d \geq d_{c r}\end{cases}$

Using Eqs. (2) and (3), the received power is expressed as:

$P_{r}= \begin{cases}\epsilon_{f s p} R \phi & \text { if } d<d_{c r} \\ \epsilon_{t r p} R \varphi & \text { if } d \geq d_{c r}\end{cases}$

The parameters $\epsilon_{f s p}$ and $\epsilon_{\text {trp }}$ are determined by setting Eq. (13) equals to the received power threshold $\left(P_{r}^{(t h r)}\right)$ :

$\epsilon_{f s p}=\frac{P_{r}^{(t h r)}}{R \phi}$

$\epsilon_{t r p}=\frac{P_{r}^{(t h r)}}{R \varphi}$

The required transmit power $P_{t}$, as a function of the received power threshold and the distance between the transmitter and receiver, is determined by substituting Eqs. (14) and (15) in Eq. (12):

$P_{t}= \begin{cases}\frac{P_{r}^{(t h r)} d^{2}}{\phi} & \text { if } d<d_{c r} \\ \frac{P_{r}^{(t h r)} d^{4}}{\varphi} & \text { if } d \geq d_{c r}\end{cases}$

The receiver threshold $P_{r}^{(t h r)}$ is determined by estimating the noise at the receiver. Considering the thermal noise floor equals to $99 \mathrm{dBm}$, the receiver noise figure of $17 \mathrm{~dB}$ and the required signal-to-noise ratio (SNR) of at least $30 \mathrm{~dB}$ to receive the signal with no errors [42], the minimum received power must be at least $\left(P_{r}^{(t h r)}=-52 \mathrm{dBm}=6.3 \mathrm{nW}\right)$ for successful reception.

Considering the experiment parameters $\left(G_{t}=G_{r}=1, h_{t}=h_{r}=\right.$ $1 \mathrm{~m}, \lambda=0.124 \mathrm{~m}$ ) [43], and as the IEEE 802.15.4 standard targets a 


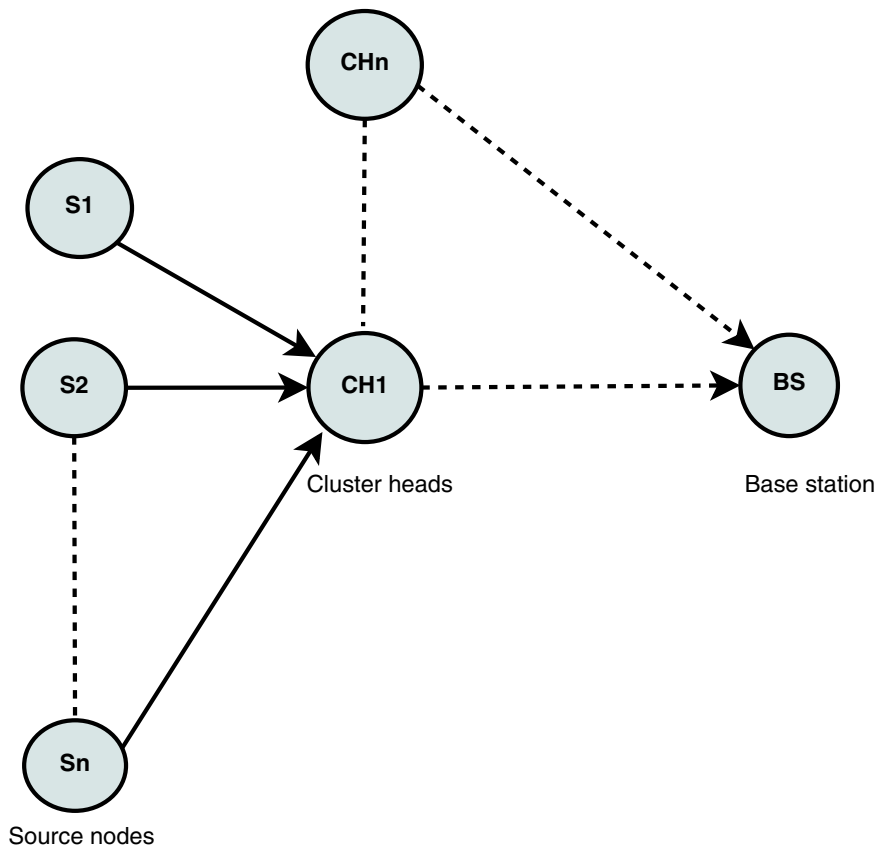

Fig. 1. Cluster-based network model.

bit rate of $R=250 \mathrm{kbps}$, the Eqs. (14) and (15) are expressed as:

$\left\{\begin{array}{l}\epsilon_{f s p}=2.59 \times 10^{-10} \mathrm{~J} / \mathrm{bit} / \mathrm{m}^{2} \\ \epsilon_{t r p}=2.52 \times 10^{-14} \mathrm{~J} / \mathrm{bit} / \mathrm{m}^{4}\end{array}\right.$

\subsection{System model}

We consider a clustered WSN system, in which a set $\mathcal{N}$ of sensor nodes with $|\mathcal{N}|=N$ are distributed in a given area and clustered in $C$ clusters with $j \in \mathcal{C} \triangleq\{1 \ldots C\}$ cluster heads. The clustered sensor network contains $(N-C=S)$ non- $\mathrm{CH}$ nodes such that $i \in \mathcal{S} \triangleq\{1 \ldots S\}$ which present the source nodes of the network that are attached to the cluster heads. The selected source nodes sense the environment and forward the sensed data to elected $\mathrm{CHs}$. After performing data aggregation, the $\mathrm{CH}$ nodes transmit data to the base station. Therefore, the transmission system is decomposed into three main and possible scenarios:

- $\mathcal{T}_{\mathcal{S}} \rightarrow \mathcal{T}_{\mathcal{C}}$ : Source nodes exchange their information with their near attached $\mathrm{CHs}$. Each node is connected via a direct available link to the nearest $\mathrm{CH}$.

- $\mathcal{T}_{\mathcal{C}} \rightarrow \mathcal{T}_{B S}$ : After receiving the packets, the $\mathrm{CH}$ combines the incoming streams and retransmits them to the base station. The base station is linked with $\mathrm{CHs}$ via direct channel.

- $\mathcal{T}_{\mathcal{S}} \rightarrow \mathcal{T}_{B S}$ : Source nodes convey directly their data to the base station when no $\mathrm{CH}$ is selected or the BS is closer to the source node than to the $\mathrm{CH}$.

Fig. 1 shows a simple model of the clustering and transmission scenarios. Considering a clustered network system with $C$ cluster heads and $S$ source nodes, the energy consumed by all the network nodes during a round $r, E_{\text {Net }}^{(r)}$ is determined by:

$E_{\text {Net }}^{(r)}=\sum_{i=1}^{S} E_{i}^{(r)}+\sum_{j=1}^{C} E_{j}^{(r)}$

Where $E_{i}^{(r)}$ and $E_{j}^{(r)}$ are the energies dissipated in radio and computational processes in each source node $i \in \mathcal{S}$ and cluster head $j \in \mathcal{C}$ at each round $r$, respectively.

The total energy of a cluster head $j$ at each round is expressed as the sum of energies of reception, aggregation, error controlling and transmitting to the base station:

$$
E_{j}^{(r)}=E_{j, R x}^{(r)}+E_{j, E C C}^{(r)}+E_{j, A g g}^{(r)}+E_{j,(T x)_{\text {toBS }}}^{(r)}
$$

Where

$E_{j,(T x)_{\text {toBS }}^{(r)}}^{(r)}= \begin{cases}k\left(E_{c k t}^{(r)}+\epsilon_{f s p} d_{j_{\text {toBS }}}^{2}\right) & \text { if } d_{j_{\text {toBS }}}<d_{c r} \\ k\left(E_{c k t}^{(r)}+\epsilon_{t r p} d_{j_{\text {toBS }}}^{4}\right) & \text { if } d_{j_{\text {toBS }} \geq d_{c r}}\end{cases}$

and

$$
\left\{\begin{array}{l}
E_{j, R x}^{(r)}=\left|s_{j}^{(r)}\right| k E_{c k t}^{(r)} \\
E_{j, A g g}^{(r)}=\left|s_{j}^{(r)}\right| k E_{D A}^{(r)} \\
E_{j, E C C}^{(r)}=\left|s_{j}^{(r)}\right| k E_{E C C}^{(r)}
\end{array}\right.
$$

Where $E_{j,(T x)_{t o B S}}^{(r)}$ is the energy consumed when a cluster head $j$ transmits data to $\mathrm{BS}, E_{j, R x}^{(r)}$ is the energy consumed when a cluster head receives data from its cluster's nodes, $E_{j, A g g}^{(r)}$ is the energy used by $\mathrm{CH}$ node of cluster $j$ to aggregate data, $E_{D A}^{(r)}$ is the energy consumed by $\mathrm{CH}$ to fuse 1 bit data, $\left|s_{j}^{(r)}\right|$ is the number of source nodes attached to a cluster head $j$ in a round $r, E_{j, E C C}^{(r)}$ refers to the energy consumed by each $\mathrm{CH}$ to control the errors of transmission and $d_{j_{\text {toBS }}}$ is the distance between each cluster head and BS.

According to the network density and inter-node distances, some $\mathrm{CH}$ nodes may operate on the free space mode while others may operate on multipath mode. By assuming that $f$ is the free space operating $\mathrm{CH}$ nodes, the transmission energy of $\mathrm{CH}$ is expressed as follow:

$\sum_{j=1}^{C} E_{j,(T x)_{\text {toBS }}}^{(r)}=k\left(C E_{c k t}^{(r)}+\epsilon_{f s p} \sum_{j=1}^{f} d_{j_{\text {toBS }}}^{2}+\epsilon_{t r p} \sum_{j=1}^{C-f} d_{j_{\text {toBS }}}^{4}\right)$

Cluster heads in the network consist of $C$ transmissions, $S$ receptions, $N$ aggregations and $N$ computations. Thus, the total energy consumed by all $\mathrm{CH}$ nodes at each round in the network is:

$$
\sum_{j=1}^{C} E_{j}^{(r)}=k\left(N\left(E_{c k t}^{(r)}+E_{D A}^{(r)}+E_{E C C}^{(r)}\right)+\epsilon_{f s p} \sum_{j=1}^{f} d_{j_{\text {toBS }}}^{2}+\epsilon_{\text {trp }} \sum_{j=1}^{C-f} d_{j_{\text {toBS }}}^{4}\right)
$$

As the clustered network contains $S$ non- $\mathrm{CH}$ nodes, some of them may operate on free space mode while others may operate on multipath mode. Let $f_{1}$ the number of source nodes operating on the free space mode, the energy of the non- $\mathrm{CH}$ nodes is:

$\sum_{i=1}^{S} E_{i}^{(r)}=k\left(S E_{c k t}^{(r)}+\epsilon_{f s p} \sum_{i=1}^{f_{1}} d_{i, j}^{2}+\epsilon_{t r p} \sum_{i=1}^{S-f_{1}} d_{i, j}^{4}\right)$

From Eqs. (23) and (24), we expose the total energy consumed by $\mathrm{CHs}$ and their attached source nodes during a round in the clustered sensor network as follow:

$$
\begin{aligned}
E_{\text {Net }}^{(r)}= & k\left((N+S) E_{c k t}^{(r)}+N\left(E_{D A}^{(r)}+E_{E C C}^{(r)}\right)+\epsilon_{f s p}\left(\sum_{j=1}^{f} d_{j_{\text {toBS }}}^{2}+\sum_{i=1}^{f_{1}} d_{i, j}^{2}\right)\right. \\
& \left.+\epsilon_{\text {trp }}\left(\sum_{j=1}^{C-f} d_{j_{\text {toBS }}^{4}}^{4}+\sum_{i=1}^{S-f_{1}} d_{i, j}^{4}\right)\right)
\end{aligned}
$$

\section{Error control coding for WSN}

Critical wireless sensor applications such as environment monitoring require the delivery of high priority events in a reliable and faster manner without any loss on the path from sensor nodes to the base station. However, the unstable nature of channels is a critical and unpredictable factor which disturbs the signal and causes 
data corruption [46]. All these constraints emphasize the need for reliability and robustness of the data transport and minimization of errors probability in spite of flawed channels [47]. Therefore, error control coding is a key solution for effective communications in WSN.

\subsection{ARQ scheme}

The ARQ scheme is one of the paramount controlling protocols used in WSN due to its simplicity and efficiency in error-free environment. This protocol consists of analyzing the packets and detecting the errors induced by the channel. The receiver is susceptible to inform the sender by sending a positive acknowledgment (ACK) if the packets are correctly received while sending a negative acknowledgment (NACK) if the packets are received with errors or lost in the channel. This protocol is typically based on the powerful Cyclic Redundancy Check (CRC) code which is characterized by a polynomial generator $(G(x))$ to generate the parity check bits at the sender and to detect the errors at the receiver. The CRC is transmitted as a header of packet and used at the receiver to verify the integrity of the received packets.

We consider the simple case of single hop send and wait ARQ between each source $(i \in \mathcal{S})$ and its attached cluster head $(j \in \mathcal{C})$. The total energy dissipated to transmit data by using ARQ protocol in a round $r$ is defined as:

$E_{A R Q}^{(r)}=E_{i}^{(r)}+E_{j}^{(r)}$

Where $E_{i}^{(r)}$ and $E_{j}^{(r)}$ are the energies consumed at the sender (source node) and destination ( $\mathrm{CH}$ node), respectively. These energies can be defined by:

$\left\{\begin{array}{l}E_{i}^{(r)}=(\gamma+1)\left(E_{T_{x_{\text {Data }}}^{(r)}}^{(r)}+E_{R x_{A C K / N A C K}}^{(r)}\right) \\ E_{j}^{(r)}=(\gamma+1)\left(E_{R x_{\text {Data }}}^{(r)}+E_{T x_{A C K / N A C K}}^{(r)}\right)\end{array}\right.$

Where $E_{T x_{\text {Data }}}^{(r)}$ is the energy consumed by source nodes to transmit their sensed and coded data (Payload $+C R C), E_{R x_{\text {Data }}}^{(r)}$ is the energy consumed by $\mathrm{CH}$ nodes to receive the transmitted data, $E_{T x_{A C K / N A C K}}^{(r)}$ is the energy consumed by $\mathrm{CH}$ nodes to transmit a positive or negative acknowledgment, $E_{R x_{A C K / N A C K}}^{(r)}$ is the energy dissipated by source nodes to receive the acknowledgment packets and $\gamma$ is the number of possible retransmissions ( $\gamma=0$ when a packet is correctly received).

The source node transmits the data (Payload $+C R C)$ to the $\mathrm{CH}$ and receives an $A C K / N A C K$ packet from the $\mathrm{CH}$ node. If the receiver detects an error in the received packet, a NACK must be transmitted to the sender in order to retransmit the packet.

\subsection{FEC scheme}

In FEC approach, errors are both detected and corrected at the receiver end. To enable the receiver to correct data, Certain redundancy is combined with the original information according to a coding algorithm at the transmitter. The capability of correcting errors induced by flawed channels in the received sequence is measured by providing better BER performance for the same SNR compared to uncoded scheme or by providing the same BER at lower SNR than uncoded scheme [10]. The coding gain is the difference in required SNR to achieve a certain BER for a given coding scheme compared to uncoded scheme. Efficient coding schemes are characterized by higher coding gain and less transmission energy. In this work, we consider LDPC codes as FEC scheme to protect the sensed data, as they feature eminent performance over other codes $[27,30]$.

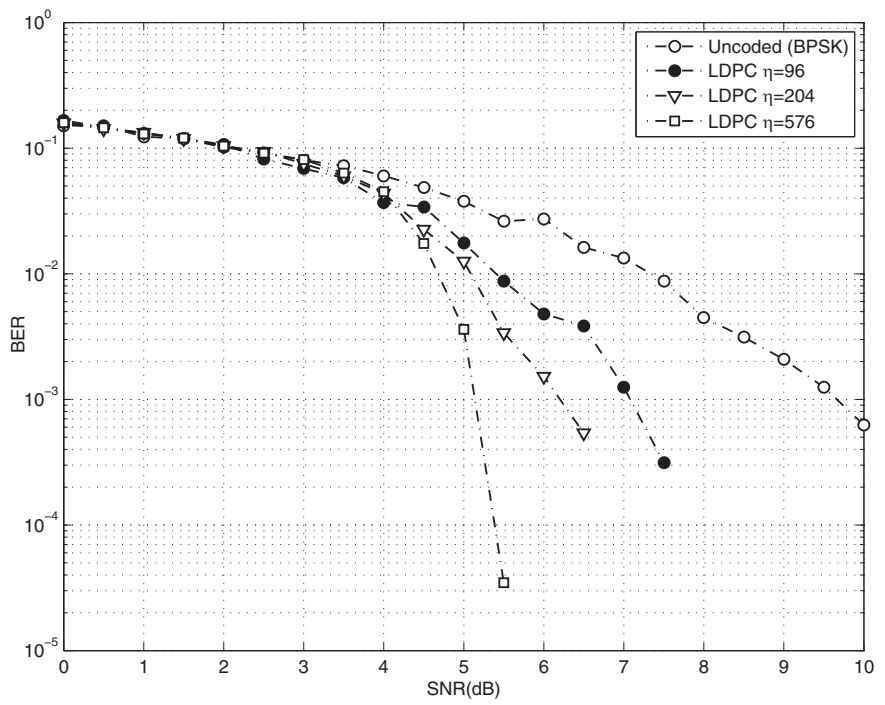

Fig. 2. BER simulation of LDPC scheme with different coding length $\eta$.

LDPC codes are linear error correcting block codes described by a sparse $(M \times N)$ parity-check matrix $H$ [48]. This matrix can be represented as a bipartite graph known as Tanner Graph [49], with $M$ check nodes and $N$ variable nodes. Ones in the parity-check matrix $H$ are presented by edges in Tanner graph and most of decoding algorithms require the exchange of information along the edges of the graph. Belief Propagation is the common algorithm used to decode LDPC codes due to its performance [50]. The BP algorithm emphasizes that variable nodes update the received information from the channel according to the parity check equations evaluated at the check nodes. This process is repeated several times until the maximum number of iterations is reached.

The complexity of LDPC decoding increases with either increasing the decoding iterations or increasing the codeword length $\eta$ which requires significant amount of energy resources. Moreover, the amount of sensed and exchanged data by sensor nodes in WSN applications is typically limited. In this context, we considered short block length FEC codes for error correction in WSN.

Fig. 2 shows the BER performance of LDPC coding scheme with various coding lengths under BPSK modulation and AWGN channel. We considered three regular LDPC codes with the same coding rate $(\tau=0.5)$, a number of iterations $(i t=50)$ and with different coding length $\left(\eta_{1}=96, \eta_{2}=204\right.$ and $\left.\eta_{3}=576\right)$. The performed simulation reveals that increasing the coding length enhances the performance of the LDPC coding scheme by raising the coding gain. This gain provided by the coding scheme represents a transmission energy saving such that transmitting a coded data requires less transmit power than transmitting uncoded data. In the other hand, the complexity of decoding process increases by raising the coding length $\eta$. In another context, Fig. 3 displays a BER simulation of LDPC scheme with the same coding length $(\eta=96)$ and various decoding iterations of $\left(i t_{1}=5, i t_{2}=10\right.$, and $\left.i t_{3}=50\right)$. It is shown that increasing the number of decoding iterations clearly improves the reliability by increasing the coding gain from $2 \mathrm{~dB}$ with 5 iterations to $3 \mathrm{~dB}$ with 10 iterations and $3.5 \mathrm{~dB}$ with 50 iterations when targeting a BER of $10^{-3}$. Nevertheless, the computation energy of decoding increases by requiring more iterations to correctly decode the data. If the additional cost of the decoding energy at the receiver overcomes the transmit energy saving, LDPC coding process would deplete nodes energy instead of saving energy. 


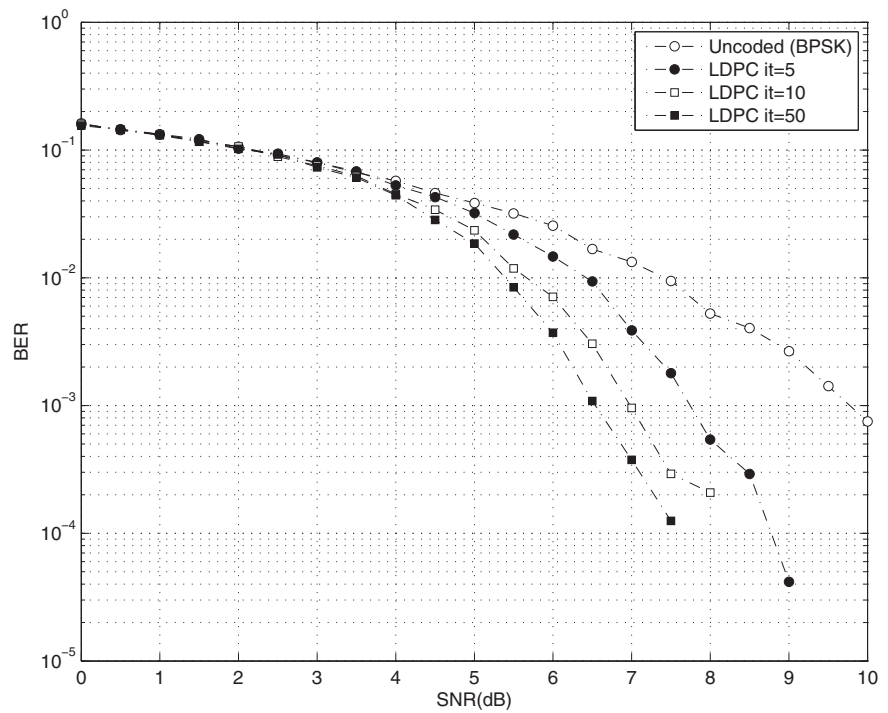

Fig. 3. BER simulation of LDPC scheme with different decoding iterations.

The total computational energy dissipated due to using FEC scheme in a round $r$ is defined as:

$E_{\text {comp }}^{(r)}=k\left(E_{\text {cod }}^{(r)}+E_{d e c}^{(r)}\right)$

Where, $E_{c o d}^{(r)}$ and $E_{d e c}^{(r)}$ are the computation energies required for coding and decoding processes, respectively.

In the case of block codes, encoding is typically a simple process with insignificant computational energy [12]. Thus, the energy depleted in encoding is not considered in this work $\left(E_{\text {cod }}=0\right)$. Moreover, the decoding energy is computed by counting the number of addition, multiplication, division and comparison executed at nodes.

The decoding energy associated with BP algorithm of LDPC codes in each iteration is given as [51,52]:

$E_{L D P C}^{d e c}=\prod_{k=1}^{n}(3 \eta \hat{i}+6 \sigma \eta \hat{j}-10 \eta) \varepsilon_{m u l t}+(3 \eta \hat{j}+\eta) \varepsilon_{a d d}$

Where $\hat{i}$ and $\hat{j}$ are the weights of row and column of parity check matrix of LDPC code, $\sigma$ is the correction capacity, $n$ is the number of optimal iterations correspond to viable BER. $\varepsilon_{\text {add }}$ and $\varepsilon_{\text {mult }}$ denote the energies consumption per $m$-bit in the addition and multiplication, respectively, of field elements in Galois Field $\left(G F\left(2^{m}\right)\right)$. The values of these energies are computed in [51] for $0.18 \mu \mathrm{m}, 2.5 \mathrm{~V}$ CMOS based implementation.

\section{Proposed adaptive coding frameworks}

In WSNs, the energy efficiency and reliability vary with different network architectures and topologies. Cluster-based routing provides a promising solution for communications and presents a pivotal approach to lower the energy consumption and maximize the WSN lifetime. However, the CHs in a clustered sensor network are the most consuming nodes of energy due to the fact that they perform many paramount computational and communication operations. Each $\mathrm{CH}$ in the network is a source node that collects measurements from the environment. At the same time, it serves as a relay by receiving different packets, decoding, aggregating, encoding and then transmitting them to the base station. In presence of a complex coding and decoding schemes, these processes may have the worst effect on energy depletion.

Fig. 4 shows a comparison between energy depletion of clustering transmission when using decoding at the $\mathrm{CH}$ and when the



Fig. 4. Total energy of clustered network with and without decoding at $\mathrm{CH}$.



Fig. 5. Total energy of the clustered network with different $\mathrm{CH}$ percentages.

decoding is carried out only at the BS. In the first case, all nodes encode their sensed data and transmit them to the CHs that aggregate the received data and transmit them to the BS where the decoding is performed. In the second case, source nodes encode their sensed data and transmit them to the attached CHs. After reception of data, $\mathrm{CHs}$ decode and correct each packet before transmission to the BS. The performed simulation clearly shows that the clustering system with decoding at $\mathrm{CH}$ intensely drains nodes energy and lowers the clustering performance compared to clustering system without decoding at $\mathrm{CH}$. Moreover, the effect of decoding on clustering system increases by raising the percentage of $\mathrm{CH}$ nodes in the network. Fig. 5 shows the total energy of clustered network with different $\mathrm{CH}$ percentages in presence of decoding. Increasing the number of selected $\mathrm{CH}$ in the network from $10 \%$ to $30 \%$ interestingly speeds up the dead of nodes and hastens the network lifetime. It is shown that more the number of $\mathrm{CH}$ is important, worse the network energy behaves, which is primarily related to the processing effects.

In this work, we proposed substantial coding frameworks for clustered sensor network which consider the propagation channel features and inter-node distance to decide adequate coding, de- 


\section{Approach 1 (FEC/FWD)}

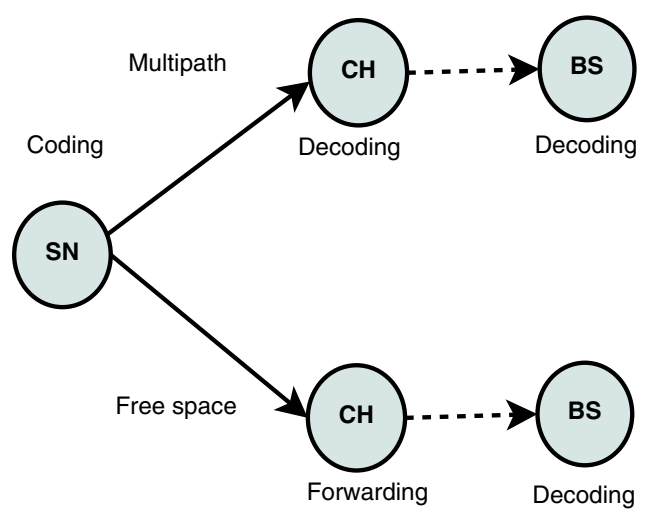

Approach 2 (FEC/ARQ)



Fig. 6. Description of the proposed coding frameworks.

coding or control usage. The attractiveness of the proposed coding frameworks reside in the capacity of $\mathrm{CH}$ nodes to avoid the decoding process if it would not be necessary based on the channel state and the distance.

At short inter-node distances, the transmission energy is comparable to the computation energy consumed in coding and decoding processes and the effects of channel conditions are often insignificant [44]. However, at large inter-node distances, the transmission energy is significantly larger than the energy consumed in coding and decoding processes. Moreover, the effect of channel impairments is important. In clustered network, source nodes are likely attached to the closest $\mathrm{CH}$ nodes, which enable the line of sight free space propagation to be mostly adopted instead of multipath propagation. Thus, the transmitted packets are relatively unaffected and resistant to channel impairments. In this case, the decoding process would not be necessary.

The base station is generally placed distantly from the network which makes the multipath propagation to be likely present. Moreover, the importance of the aggregated packets transmitted from $\mathrm{CHs}$ requires the use of ECC. At this stage, using efficient decoding algorithm at the BS turns out to be a convenient approach to protect the transmitted data. The proposed schemes take into account all the mentioned features in order to design adaptive coding schemes for clustered WSN. Fig. 6 shows a description of the proposed coding approaches.

The inter-node distances and channel state were used as parameters controlling the coding usage in WSN. During the clustering phase, all nodes in the network receive an acknowledgment from the selected $\mathrm{CHs}$ by which nodes are capable of assessing the distance from each $\mathrm{CH}$. Assuming that the distance between each sensor node and the base station is already known, each node in the network compares the distance both to the $\mathrm{CH}$ and BS. If the distance to the BS is lower than the distance to the $\mathrm{CH}$ $\left(d_{t o B S} \leq d_{t o C H}\right)$, then nodes are deemed to be communicated directly with the base station, while communicated with the $\mathrm{CH}$ node (according to clustering algorithm) in the other case. Algorithm 1 depicts the transmission phase of each source node in the network.

In this work, we considered ARQ and FEC based schemes to encode the source packets and decode/verify the received packets. In direct communications between source nodes and the base station, encoded packets are transmitted and efficiently decoded at the BS without energy constraint. If nodes should communicate with the $\mathrm{BS}$ through $\mathrm{CH}$ nodes, the source packets are decoded or verified at

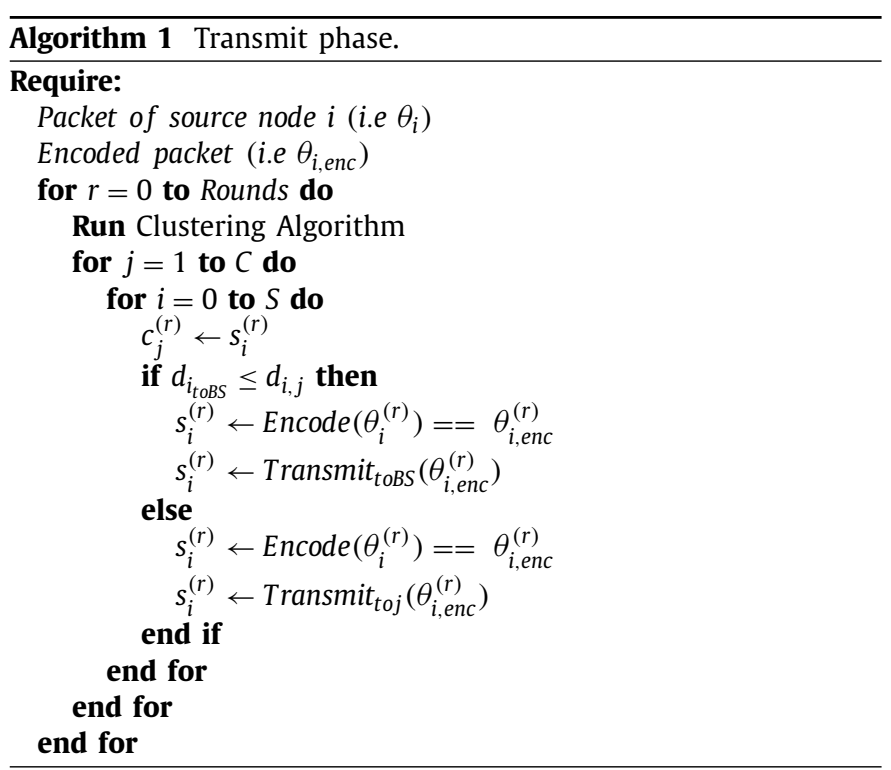

the $\mathrm{CH}$ and then at the BS. For FEC scheme, a LDPC coding scheme is selected to be used in each source node for encoding its readings by introducing some redundancy. Thus, the encoded packets in Algorithm 1 can be expressed as $\theta_{i, e n c}=\operatorname{LDPC}\left(\theta_{i}\right)$. For ARQ based scheme, a CRC overhead is included in each payload of the encoded packets $\theta_{i, e n c}=\left(\theta_{i}+C R C_{i}\right)$.

\subsection{Adaptive FEC/FWD scheme}

In the first adaptive scheme (based FEC/FWD) (Algorithm 2), the cluster heads decide of whither to correct the packets using FEC with decoding or forward the encoded packets without decoding (FWD) based on the distance and channel state. Received Signal Strength Indicator (RSSI) is used to evaluate the received signal and estimate the transmission distance. Based on the RSSI, $\mathrm{CH}$ nodes compare the estimated distance with the crossover distance to estimate the signal strength and channel state. If the channel is stated free space, the $\mathrm{CH}$ forwards the encoded packets without decoding due to the fact that the channel is considered line of sight and the readings are unaffected. In contrast, when the chan- 


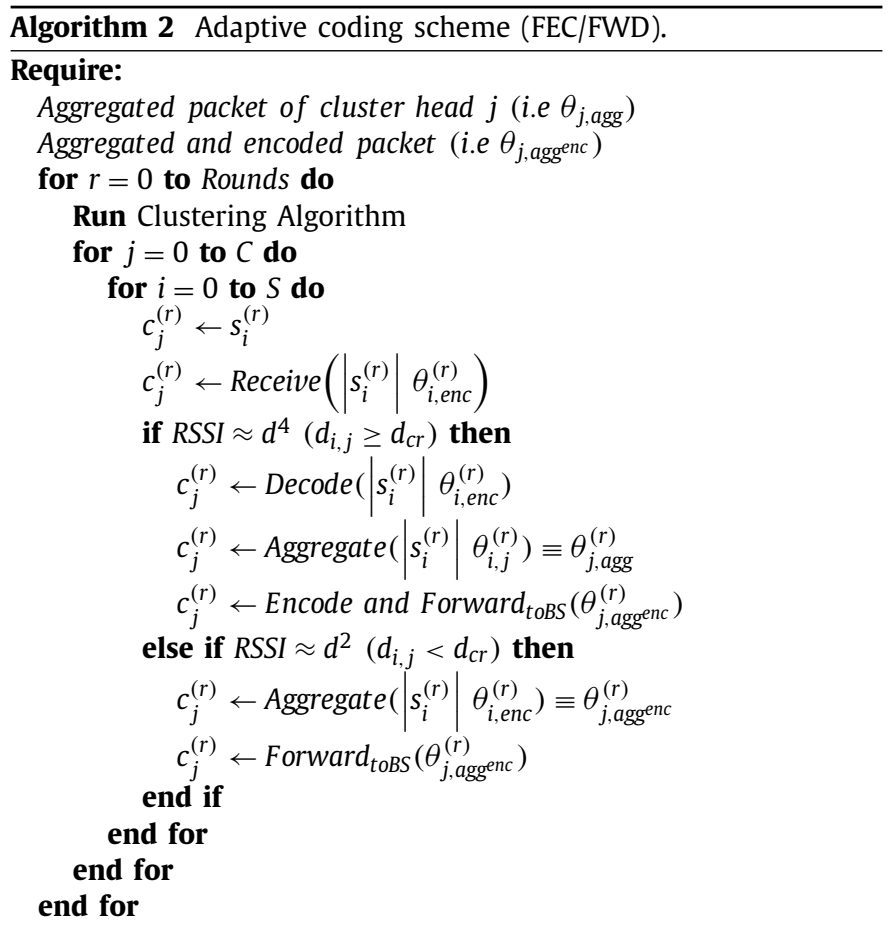

nel is stated multipath, the $\mathrm{CH}$ nodes should decode, encode and then transmit the data to the BS. In this case, the transmitted packets are corrected at the $\mathrm{CH}$ and BS in order to ensure high reliability. Note that in both cases, the packets are decoded at the base station using the iterative decoding algorithm to enable effective decoding of both packets coming from source nodes and $\mathrm{CHs}$.

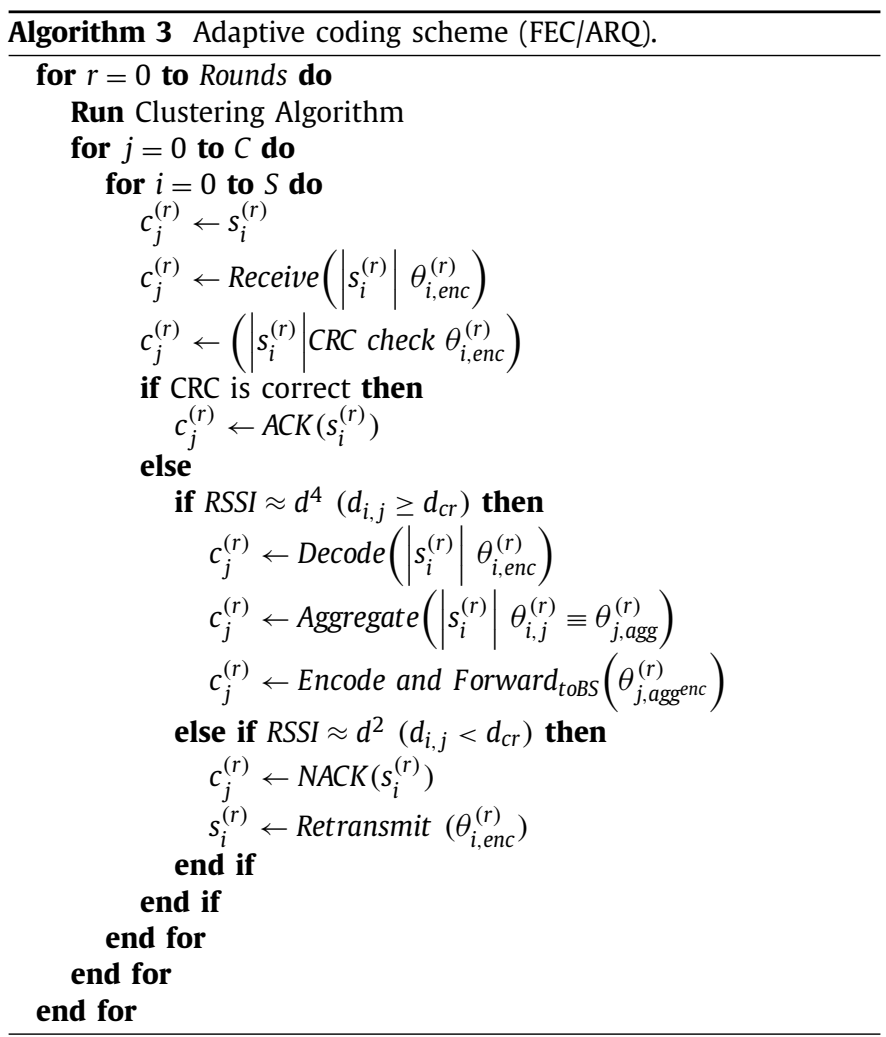

\subsection{Adaptive FEC/ARQ scheme}

In the second adaptive scheme (based FEC/ARQ) (Algorithm 3), the cluster heads decide of whither to decode the received packets or verify the integrity of packets (ACK/NACK) with ARQ. Each source node encodes its packet by using FEC scheme, and adds systematically a CRC overhead to the encoded data. Similarly to the previous scheme, RSSI is used to evaluate the received signal and estimate the transmission distance which is compared to the crossover distance to estimate the signal strength and the channel state. If the channel is a line of sight free space, the $\mathrm{CH}$ nodes verify the integrity of the received packets based on the CRC with forwarding (ACK) in the case of successful receiving and an (NACK+retransmission) in the case of an error is occurred. If the channel is stated two-ray mutipath, $\mathrm{CH}$ nodes decode, encode and transmit data to the BS. In both cases, the received packets at the BS are decoded with iterative decoding algorithm. The proposed schemes based FEC/FWD and based FEC/ARQ behave similarly in the multipath channel and differ in the free space channel.

The proposed frameworks enable to avoid the decoding process in $\mathrm{CH}$ nodes when the channel condition is line of sight and the distance is small, while applying the decoding when the channel condition is multipath. This approach aims to optimize both the energy consumption and reliability of the clustered network.

\subsection{Energy analysis}

Considering $\xi_{i}^{(r)}, \zeta_{i}^{(r)}$ and $\omega_{i}^{(r)}$ are the length of packet $\theta_{i}^{(r)}$, the length of a CRC overhead, and the length of acknowledgment of a source node $i$ in a round $r$, respectively. And considering that $s_{i, j}^{(r)}$ is a source node $i \in \mathcal{S}$ attached to the cluster head $c_{j}^{(r)} j \in \mathcal{C}$ in a round $r^{1}$, and assuming that $d_{i, j}^{(r)}=d_{j, i}^{(r)}$.

If a packet $\theta_{i}^{(r)}$ of a source node $s_{i, j}^{(r)}$ is not received correctly by the attached $c_{j}^{(r)}$ or lost in the channel, the $c_{j}^{(r)}$ transmits a $N A C K_{j, i}$ to the correspondent source node $s_{i, j}^{(r)}$ which should retransmit the packet. The energy of communicating negative acknowledgment from $c_{j}^{(r)}$ to $s_{i, j}^{(r)}$ is determined as:

$E_{(c o m)_{N A C K}}^{(r)}=\omega_{j}^{(r)}\left(2 E_{c k t}^{(r)}+\epsilon_{f s p} d_{j, i}^{2}\right)$

The energy of transmission and retransmission of a packet $\left(\theta_{i}+\right.$ $C R C_{i}$ ) from a source node $s_{i, j}^{(r)}$ is expressed as:

$E^{(r)}\left(s_{i, j}\right)_{T x+R t}=2\left(\xi_{i}^{(r)}+\zeta_{i}^{(r)}\right)\left(E_{c k t}^{(r)}+\epsilon_{f s p} d_{i, j}^{2}\right)$

As the ARQ protocol is particularly used in the free space mode, the distance is proportional to $d^{2}$.

The energy of receiving the same packet $\left(\theta_{i}+C R C_{i}\right)$ by $c_{j}^{(r)}$ from an attached $s_{i, j}^{(r)}$ after the transmission and retransmission is expressed as:

$E^{(r)}\left(c_{j}\right)_{R x}=\left(2\left(\xi_{i}^{(r)}+\zeta_{i}^{(r)}\right) E_{c k t}^{(r)}\right)$

From Eqs. (31) and (32), we express the energy of transmitting, retransmitting and receiving the same packet as:

$E_{c o m}^{(r)}=2\left(\xi_{i}^{(r)}+\zeta_{i}^{(r)}\right)\left(2 E_{c k t}^{(r)}+\epsilon_{f s p} d_{i, j}^{2}\right)$

Considering a packet $\theta_{i}$ which has to be undergo $\gamma$ retransmissions to be correctly retrieved, the Eqs. (30) and (33) are replaced with:

$E_{(\mathrm{com})_{N A C K}}^{(r)}=\omega_{i}^{(r)}\left((\gamma+1) E_{c k t}^{(r)}+\epsilon_{f s p} d_{j, i}^{2}\right)$

\footnotetext{
1 The set of source nodes attached to a cluster head $j \in \mathcal{C}$ are changed in each round $r$.
} 
$E_{c o m}^{(r)}=(\gamma+1)\left(\xi_{i}^{(r)}+\zeta_{i}^{(r)}\right)\left((\gamma+1) E_{c k t}^{(r)}+\epsilon_{f s p} d_{i, j}^{2}\right)$

When a packet of a source node $s_{i, j}^{(r)}$ is received correctly, the $c_{j}^{(r)}$ forwards an ACK to the same $s_{i, j}^{(r)}$. Assuming that ACK and NACK overheads have the same length $\zeta$, the energy of transmitting and receiving a ACK is the same of transmitting and receiving an NACK.

When no retransmission is required, the energy of transmitting a packet from $s_{i, j}^{(r)}$ to $c_{j}^{(r)}$ using ARQ is defined as:

$E^{(r)}\left(s_{i, j}\right)_{T x}=\left(\xi_{i}^{(r)}+\zeta_{i}^{(r)}\right)\left(E_{c k t}^{(r)}+\epsilon_{f s p} d_{i, j}^{2}\right)$

When using a FEC scheme, a certain number of redundancies are added to the original information. Let consider the same packet $\theta_{i}^{(r)}$ of length $\xi_{i}^{(r)}$ of a source node $s_{i, j}^{(r)}$ and its FEC encoded version $\theta_{i, e n c}^{(r)}$ with a length $\xi_{i, e n c}^{(r)} \geq \xi_{i}^{(r)}\left(\xi_{i, e n c}^{(r)}=\xi_{i}^{(r)}\right.$ when the packet is not encoded). The energy of transmitting an encoded packet is defined as:

$E^{(r)}\left(s_{i, j}\right)_{T x_{e n c}}=\xi_{i, e n c}^{(r)}\left(E_{c k t}^{(r)}+E_{e n c}^{(r)}+\epsilon_{a m p} d_{i, j}^{\alpha}\right)$

Where $\alpha$ denotes the path loss component: $\alpha=2$ for free space and $\alpha=4$ for multipath.

The energy of receiving and decoding the encoded packet at the correspondent cluster head $c_{j}^{(r)}$ is:

$E^{(r)}\left(c_{j}\right)_{R x+\operatorname{dec}}=\xi_{i, e n c}^{(r)}\left(E_{c k t}^{(r)}+E_{d e c}^{(r)}\right)$

The proposed coding approach FEC/ARQ consists of transmitting the encoded packet $\theta_{\text {enc,i }}^{(r)}$ along with the CRC payload:

$E^{(r)}\left(s_{i, j}\right)_{T x}=\left(\xi_{i, e n c}^{(r)}+\zeta_{i}^{(r)}\right)\left(E_{c k t}^{(r)}+E_{e n c}^{(r)}+\epsilon_{a m p} d_{i, j}^{\alpha}\right)$

The cluster head assesses the RSSI and estimates the pathloss $\alpha$ to decide whether to decode the packet with FEC or verify/retransmit the packet with ARQ. The receiving and computational energy of the cluster head is defined as:

$E^{(r)}\left(c_{j}\right)= \begin{cases}\left((\gamma+1)\left(\xi_{i, e n c}^{(r)}+\zeta_{i}^{(r)}\right)+\gamma \omega_{i}^{(r)}\right) E_{c k t}^{(r)}+\gamma \epsilon_{f s p} d_{j, i}^{2} & \text { if } \alpha \approx 2 \\ \left(\xi_{i, e n c}^{(r)}+\zeta_{i}^{(r)}\right)\left(E_{c k t}^{(r)}+E_{d e c}^{(r)}\right) & \text { if } \alpha \approx 4\end{cases}$

Where $\gamma$ is the number of required transmissions of ACK/NACK, with $(\gamma=0)$ when a packet is received correctly.

When using the adaptive coding scheme with (FEC/FWD), the receiving and computational energy of cluster head is expressed as:

$E^{(r)}\left(c_{j}\right)= \begin{cases}\xi_{i, e n c}^{(r)} E_{c k t}^{(r)} & \text { if } \alpha \approx 2 \\ \xi_{i, e n c}^{(r)}\left(E_{c k t}^{(r)}+E_{d e c}^{(r)}\right) & \text { if } \alpha \approx 4\end{cases}$

\section{Results and discussion}

A clustered wireless sensor network is implemented using Matlab in order to assess the performance of the adaptive coding frameworks. We select the same clustering algorithm used in LEACH protocol to generate the clusters in the network [43]. We consider a set of $N=50$ homogenous sensor nodes randomly deployed in area $\mathcal{D}$ with $x_{D}=y_{D}=50 \mathrm{~m}$ and communicate with a base station placed at $100 \mathrm{~m}$ from $\mathcal{D}$. Each round, $5 \%$ of nodes are selected to be $\mathrm{CHs}$. We assume that all nodes have the same starting energy $E_{0}$ of $0.5 \mathrm{~J}$ and the base station has unlimited energy. Each node sends a packet $\theta_{i}$ of 2000 bits to the base
Table 2

Simulation parameters.

\begin{tabular}{ll}
\hline Parameters & Value \\
\hline$G_{r}, G_{t}$ & $1 \mathrm{~dB}$ \\
$h_{t}, h_{r}$ & $1 \mathrm{~m}$ \\
$N$ & 50 \\
$\delta$ & $2000 \mathrm{bits}$ \\
$f_{c}$ & $2.4 \mathrm{GHz}$ \\
$R$ & $250 \mathrm{kbps}$ \\
$E_{0}$ & $0.5 \mathrm{~J}$ \\
$E_{D A}$ & $5 \mathrm{~nJ} / \mathrm{bit} /$ signal \\
$d_{c r}$ & $101 \mathrm{~m}$ \\
$E_{\text {add }}$ & $3.3 \times 10^{-5} \mathrm{~m}(\mathrm{~mW} / \mathrm{MHz})$ \\
$E_{\text {mult }}$ & $3.7 \times 10^{-5} \mathrm{~m}^{3}(\mathrm{~mW} / \mathrm{MHz})$ \\
\hline
\end{tabular}

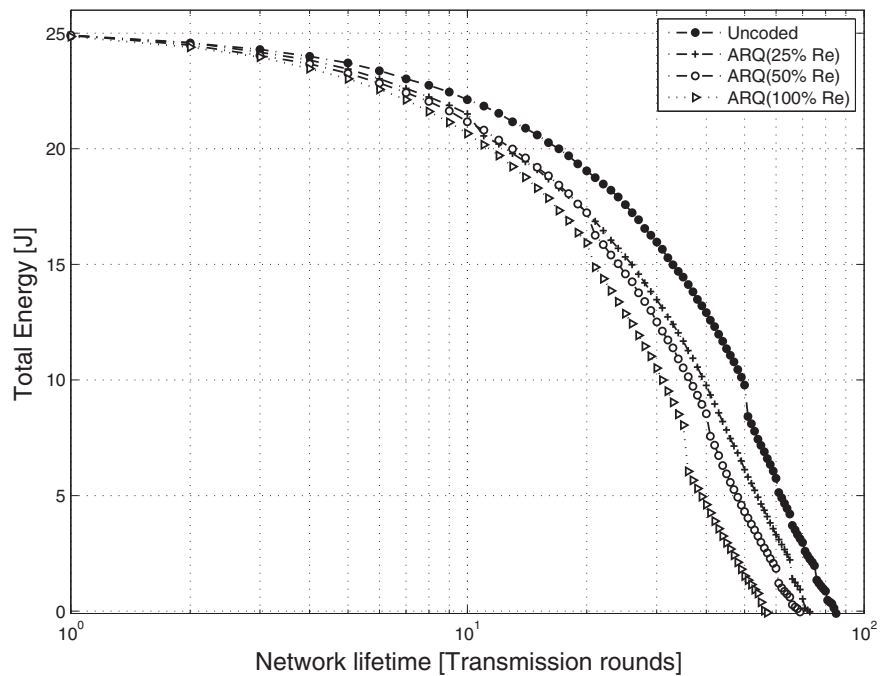

Fig. 7. Total energy of clustered network using ARQ scheme.

station directly or through $\mathrm{CHs}$ during each round. The $\mathrm{CHs}$ decode, encode, aggregate and transmit the packets to the base station. The energy dissipated for data aggregation process is set as $E_{D A}=5 \mathrm{~nJ} /$ bit/signal [43]. All the nodes transmit their data in a TDMA scheme. Table 2 depicts the radio parameters used for simulation tests.

We are interested in evaluating the total energy consumption of the network with and without coding scheme. LDPC $(\eta=96)$ and CRC-16 are used in the simulation for FEC and ARQ schemes to evaluate the effect of coding on network energy as they feature a small codeword length and small number of redundancies. Fig. 7 shows the total system energy of clustered sensor network for the uncoded scheme and ARQ based schemes in $50 \mathrm{~m}^{2}$ sensor network. It is clearly shown that more the retransmissions are required by the $\mathrm{CH}$ more the energy is depleted. In fact, the $A R Q(100 \% R e)$ scheme when all the received packets require retransmissions is the most energy consuming compared to $A R Q(50 \%$ $R e)$ and $A R Q(25 \% R e)$ schemes when $50 \%$ and $25 \%$ of packets require retransmissions, respectively. However, in line of sight free space environment where the inter-node distances are significantly small, it is likely that the channel is not immensely affected. Thus, in this work, we assumed that only up to $50 \%$ of retransmissions are possibly required in free space propagation.

In Figs. 8 and 9, we plot the total energy of the clustered network with uncoded, FEC and ARQ schemes in both $50 \mathrm{~m}^{2}$ and $100 \mathrm{~m}^{2}$ sensor network. Fig. 8 shows that FEC based scheme is the most energy consuming compared to the ARQ based scheme even though $50 \%$ of packets require retransmissions. This is primarily refers to the decoding energy and its effect on $\mathrm{CH}$ nodes. However, when increasing the inter-node distances in $100 \mathrm{~m}^{2}$ sen- 


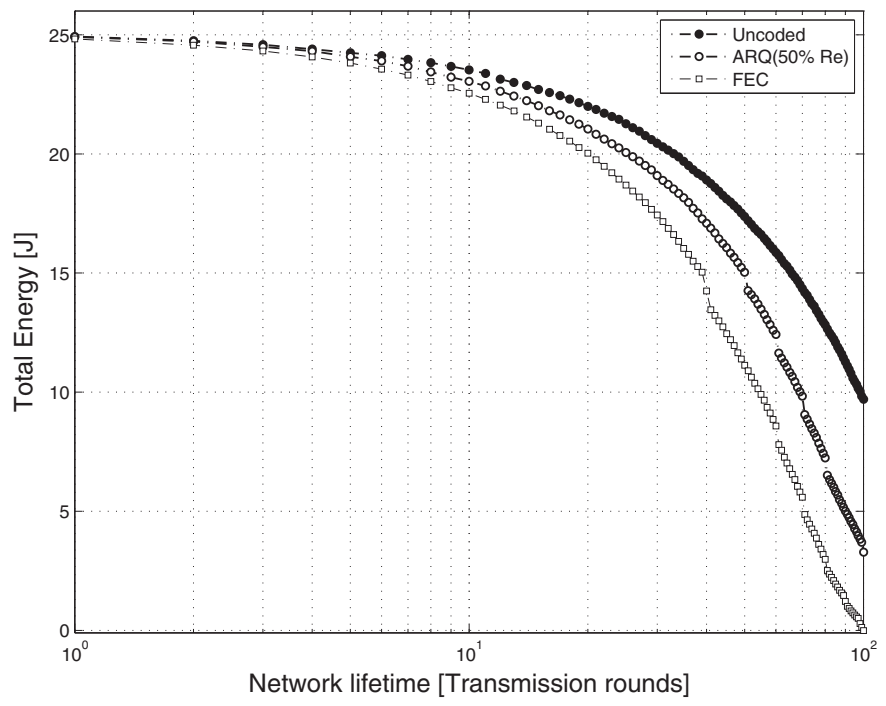

Fig. 8. Total energy of clustered network using ARQ and FEC schemes in $50 \mathrm{~m}^{2}$ network.

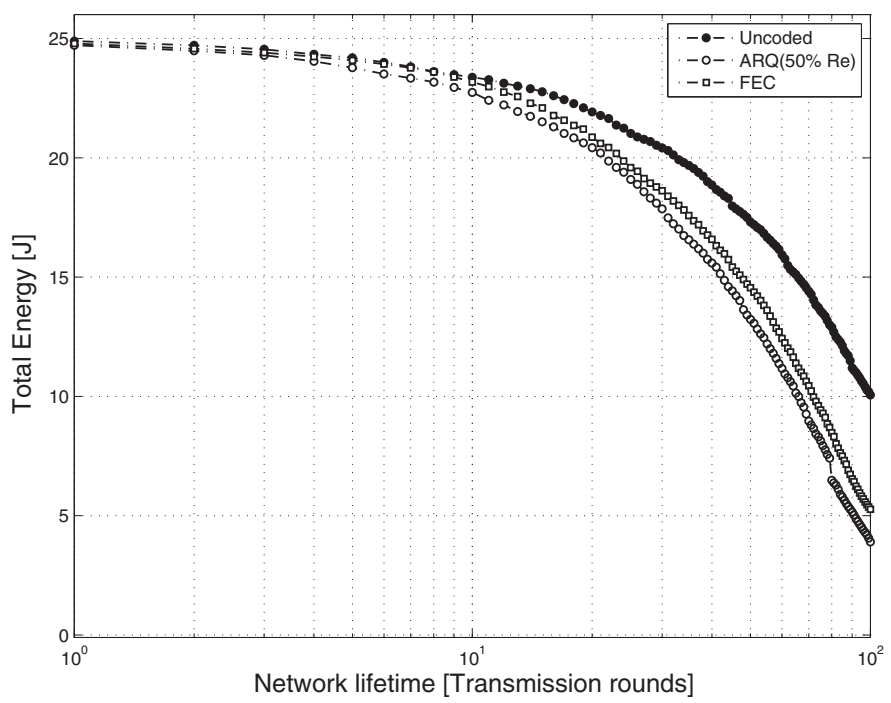

Fig. 9. Total energy of clustered network using ARQ and FEC schemes in $100 \mathrm{~m}^{2}$ network.

sor network (Fig. 9), the ARQ scheme interestingly consumes more than FEC scheme which is due to the excessive transmission energy. Therefore, we come up with these conclusions:

- Using a strong coding scheme with complex decoding algorithm in cluster heads is clearly not the optimized choice for energy consumption, particularly in short distances.

- Using ARQ based scheme in large inter-node distance and lossy environment is not the prime solution since the number of retransmissions will intensely drain the cluster head energy.

- In free-error environment and small distances, ARQ based scheme may have the best performance for countering the errors and saving energy.

Typically, wireless sensors are arbitrary deployed in unpredictable environment. In this case, sensor nodes are not always uniformly distributed in the network. Thus, the distance between the nodes can vary from line of sight direct distance to far multipath distance. Therefore, a practical adaptive coding scheme is mandatory in these kind of networks to ensure both the reliability and the energy efficiency. Fig. 10 shows the energy perfor-

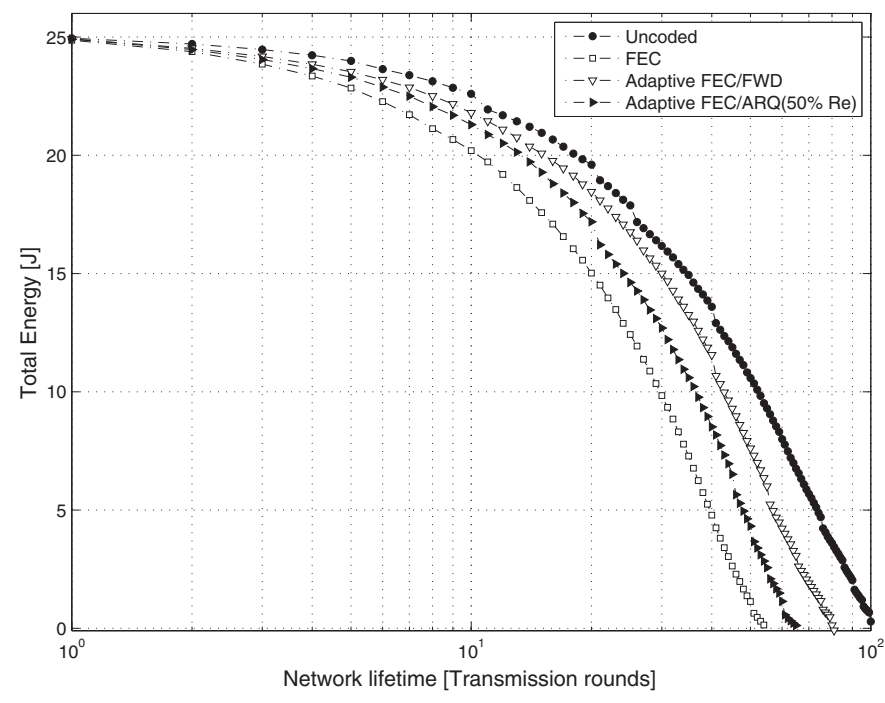

Fig. 10. Energy performance in $50 \mathrm{~m}^{2}$ clustered network.

mance of the adaptive coding frameworks based FEC/FWD and FEC/ARQ compared to FEC scheme. Table 3 summarizes the number of rounds when the first node (FND), half of nodes (HND) and last node (LND) are dead in the network. Both the proposed adaptive schemes FEC/FWD and FEC/ARQ perform energetically better than using FEC coding scheme in the entire network. The proposed FEC/ARQ scheme improves the FND, HND and LND lifetime by 53\%, $27 \%$ and $20 \%$, respectively when compared to the FEC scheme. However, FEC/FWD scheme outperforms FEC/ARQ scheme by improving the FND, HND and LND lifetime by $66 \%, 38 \%$ and $36 \%$, respectively, when compared to the FEC scheme. The FEC/FWD scheme consumes less energy than FEC/ARQ scheme by mitigating the effect of decoding on $\mathrm{CH}$ nodes. The basic idea of this adaptive scheme is to encode the packets at each source node and decode these packets in the cluster heads if the inter-node distance is large and the channel is multipath, otherwise forward the packets to the base station where they will be decoded using iterative decoding algorithm. The features of this scheme make it a salient coding framework in small sensor network with small inter-node distances.

In fact, for large scale sensor networks, most of the inter-node links and distances may undergo to multipath propagation. This will enable the FEC/FWD based scheme to apply the decoding each time. In this case, the FEC/FWD adaptive scheme will behave the same as using FEC in the whole network. For this reason, the adaptive FEC/ARQ scheme is proposed. This adaptive scheme overcomes the feature of forwarding in FEC/FWD by using an ARQ controlling scheme when the channel state is free space. As it is shown in Fig. 10, even though the system requires up to $50 \%$ of retransmissions, the proposed scheme FEC/ARQ turns out to be more effective than FEC scheme and saves energy. In Fig. 11, we extended the network dimension from $50 \mathrm{~m}^{2}$ to $100 \mathrm{~m}^{2}$ in order to enlarge the distances. Apparently, the proposed schemes FEC/FWD and FEC/ARQ preserve their performance and outperform energetically FEC scheme. Indeed, the FEC/ARQ scheme increases the FND, HND and LND lifetime by $8 \%, 10 \%$ and $7 \%$ respectively, while FEC/FWD increases their lifetime by $44 \%, 30 \%$ and $27 \%$ respectively, when compared to the FEC scheme. The adaptive FEC/FWD performs better than FEC/ARQ in both dimensions due to the fact that no decoding or retransmissions are required in free space propagation. However, it has been shown in Fig. 12 that more we increase the internode distance more the FEC/FWD scheme performance reduces. The FND, HND and LND occur at 6, 32 and 79 rounds, re- 
Table 3

Nodes die-off statistics in 50 and $100 \mathrm{~m}^{2}$ networks using FEC, FEC/ARQ and FEC/FWD schemes.

\begin{tabular}{|c|c|c|c|c|c|c|}
\hline \multirow{2}{*}{$\begin{array}{l}\text { Coding scheme } \\
\text { Network dimension }\end{array}$} & \multicolumn{2}{|c|}{ FEC scheme } & \multicolumn{2}{|c|}{ FEC/FWD scheme } & \multicolumn{2}{|c|}{ FEC/ARQ scheme } \\
\hline & $50 \mathrm{~m}^{2}$ & $\overline{100 \mathrm{~m}^{2}}$ & $50 \mathrm{~m}^{2}$ & $100 \mathrm{~m}^{2}$ & $50 \mathrm{~m}^{2}$ & $100 \mathrm{~m}^{2}$ \\
\hline FND (rounds) & 110 & 50 & 183 & 72 & 168 & 54 \\
\hline HND (rounds) & 188 & 122 & 259 & 159 & 238 & 134 \\
\hline LND (rounds) & 228 & 163 & 312 & 208 & 272 & 175 \\
\hline
\end{tabular}

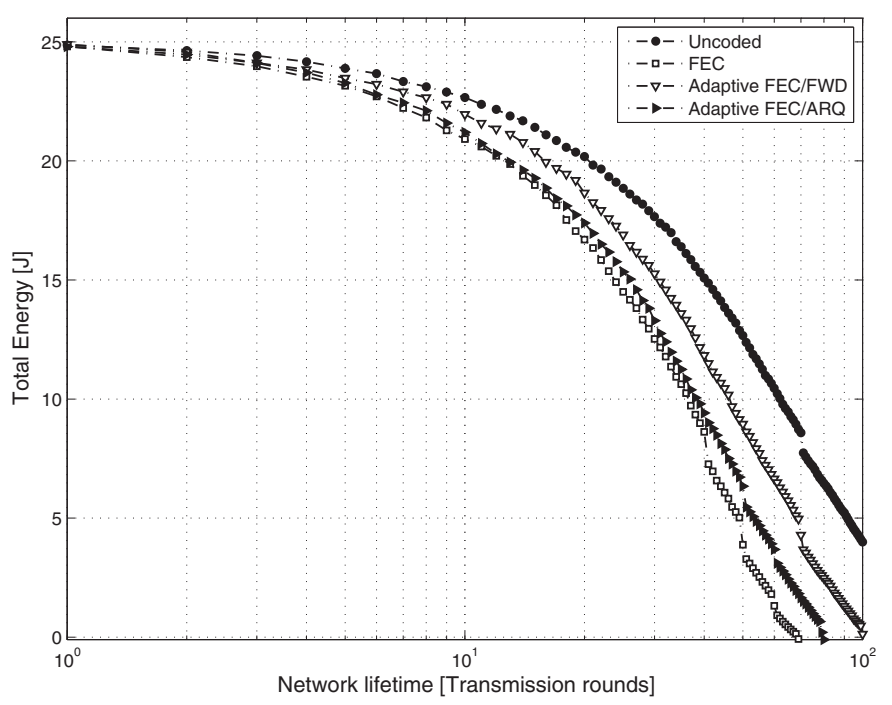

Fig. 11. Energy performance in $100 \mathrm{~m}^{2}$ clustered network.

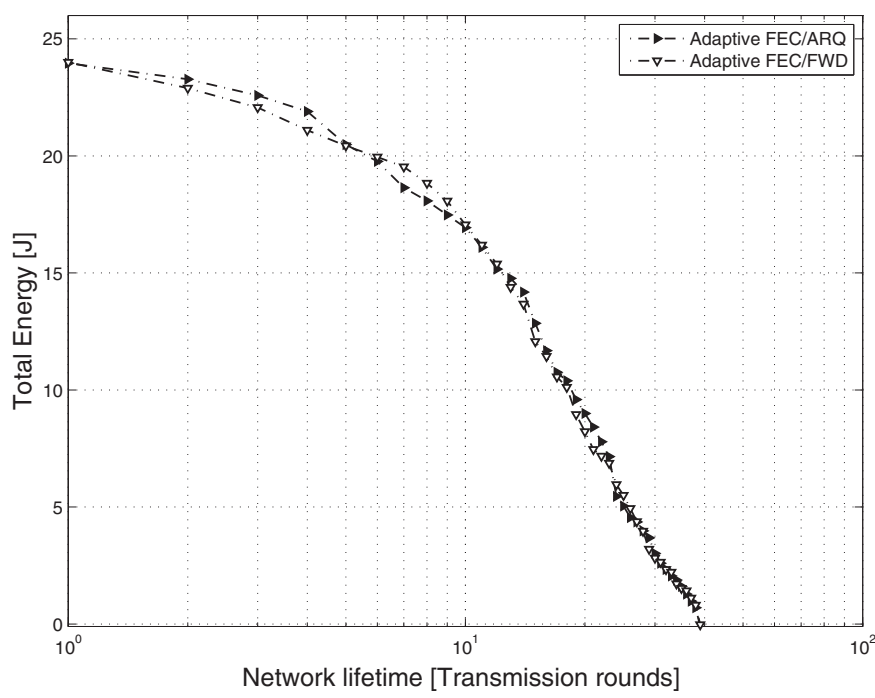

Fig. 12. Energy performance of proposed FEC/FWD and FEC/ARQ schemes in 200 $\mathrm{m}^{2}$ clustered network.

spectively, for FEC/ARQ scheme and closely occur at 5, 34 and 79 rounds, respectively, for the FEC/FWD scheme. This is substantially refers to the increase of multipath links in the networks. This automatically allows the FEC/FWD to enormously apply the decoding in $\mathrm{CH}$ nodes and increase the energy consumption. The additional cost of the FEC/ARQ compared to FEC/FWD is compensated by improving reliability when the free space links are not $100 \%$ errorfree.

\section{Conclusion}

In this paper, we investigated the coding effect in clustered WSN and proposed two substantial adaptive coding approaches for reliable and energy efficient WSN. The proposed coding frameworks take into account the channel state and the inter-node distances to decide efficiently the adequate coding usage in order to mitigate the decoding energy effects and minimize the energy consumption of sensor nodes. Both the adaptive coding approaches based on FEC/FWD and FEC/ARQ have proved to be energy efficient compared to using FEC or ARQ in the entire network. FEC/FWD scheme is particularly more suitable in small network where the inter-node distances are short and most of the links are reliable while FEC/ARQ scheme can be adopted in large networks and noisy environment where the free space propagation causes a percentage of errors. The simulation results validate the energy efficiency of the proposed coding frameworks in clustered sensor networks.

\section{References}

[1] H. Karl, A. Willing, Protocols and Architectures for Wireless Sensor Networks, Wiley-Interscience, 2007.

[2] A.H.A. Fahmy, Wireless sensor network applications, in: R.A. Lewin (Ed.), Wireless Sensor Networks, Springer, Singapore, 2016, pp. 69-213.

[3] K. Tornai, A. Olah, J. Levendovszky, Monitoring scheme for event and danger detection in wireless sensor networks, Ad Hoc. Sensor Wirel. Netw. 30 (2016) $145-161$.

[4] H.M. Ammari, S.K. Das, Centralized and clustered coverage protocols for wireless sensor networks, IEEE Trans. Comput. 61 (2012) 118-133.

[5] S. Yadav, R.S. Yadav, A review on energy efficient protocols in wireless sensor networks, Wireless Netw. 22 (2015) 335-350.

[6] N.A. Pantazis, D.J. Vergados, D.D. Vergados, C. Douligeris, Energy efficiency in wireless sensor networks using sleep mode TDMA scheduling, Ad Hoc Netw. 7 (2009) 322-343.

[7] G. Anastasi, M. Conti, M.D. Francisco, A. Passarella, Energy conservation in wireless sensor networks: a survey, Ad Hoc Netw. 7 (2008) 537-568.

[8] D.W. Lee, J.H. Kim, High reliable in-network data verification in wireless sensor networks, Wirel. Pers. Commun. 54 (2010) 501-519.

[9] M.A. Mahmood, W.K.G. Seah, I. Welch, Reliability in wireless sensor networks: a survey and challenges ahead, Comput. Netw. (Elsevier) 79 (2015) 166-187.

[10] S.L. Howard, C. Schlegel, K. Iniewski, Error control coding in low-power wireless sensor networks: when is ECC energy-efficient? EURASIP J. Wirel. Commun. Netw. 2006 (2006) 1-14.

[11] T. Elshabrawy, Network throughput analysis of IEEE 802.15.4 enabled wireless sensor networks with FEC coding under external interference, Int. J. Electron.Commun. (AEU) 69 (2015) 1641-1649.

[12] M.C. Vuran, I.F. Akyildiz, Error control in wireless sensor networks: a cross layer analysis, IEEE/ACM Trans. Networking 17 (2009) 1186-1199.

[13] N. Abughalieh, K. Steenhaut, A. Nowe, A. Anpalagan, Turbo codes for multihop wireless sensor networks with decode-and-forward mechanism, EURASIP J. Wirel. Commun. Netw 2014 (2014) 1-13.

[14] S. Manfredi, Reliable and energy-efficient cooperative routing algorithm for wireless monitoring systems, IET Wirel. Sens. Syst. 2 (2012) 128-135.

[15] D. Wei, Y.J. Vural, S. Moessner, K.R. Tafazolli, An energy-efficient clustering solution for wireless sensor networks, IEEE Trans. Wirel. Commun. 10 (2011) 3973-3983.

[16] K. Dilip, Performance analysis of energy efficient clustering protocols for maximising lifetime of wireless sensor networks, IET Wirel. Sens. Syst. 4 (2014) 9-16.

[17] K.B. Payal, K. Krishna, Leach-MAC: a new cluster head selection algorithm for wireless sensor networks, Wirel. Netw. 22 (2016) 49-60.

[18] N. Javaid, M.B. Rasheed, M. Imran, An energy-efficient distributed clustering algorithm for heterogeneous wsns, EURASIP J. Wirel. Commun. Netw. 2015 (2015) 1-11.

[19] D. Wei, Y. Jin, S. Vural, R. tafazolli, An energy-efficient clustering solution for wireless sensor network, IEEE Trans. Wireless Commun. 10 (2011) 3973-3983. 
[20] H. Gupta, S. RAO, A. Yadav, T. Dutta, Geographic routing in clustered wireless sensor networks among obstacles, IEEE Sens. J. 15 (2015) 2984-2992.

[21] D. Incebacak, R. Zilan, B. Tavli, J.M. Barcelo-Ordinas, J. Garcia-Vidal, Optimal data compression for lifetime maximization in wireless sensor networks operating in stealth mode, Ad Hoc Netw. 24 (2015) 134-147.

[22] M.Y. Naderi, R.H. Rabiee, M. khansari, M. Salehi, Error control for multimedia communications in wireless sensor networks: a comparative performance analysis, Ad Hoc Netw. 10 (2012) 1028-1042.

[23] M.E. Pellenz, R.D. Souza, M.S.P. Fonseca, Error control coding in wireless sensor networks, J. Telecommun. Syst. 44 (2010) 61-68.

[24] A.D.G. Biroli, M. Martina, G. Masera, An LDPC decoder architecture for wireless sensor network applications, Sensors J. 12 (2012) 1529-1543.

[25] L. Liang, R. Maunder, B. Al-Hashimi, L. Hanzo, A low-complexity turbo decoder architecture for energy-efficient wireless sensor networks, IEEE Trans. Very Large Scale Integr.(VLSI) Syst. 21 (2013) 14-22.

[26] H. Soude, M. Agueh, J. Mehat, Towards an optimal reed solomon codes selection for sensor networks, in: Proceedings of the 6th ACM Symposium on Performance Evaluation of Wireless Ad Hoc, Sensor, and Ubiquitous Networks, ACM Press, 2009, pp. 165-166.

[27] I. Ez-zazi, A. Eloualkadi, M. Arioua, Y. Elassari, Performance analysis of efficient coding schemes for wireless sensor networks, in: Proceedings of IEEE international Workshop on RFID and Wireless Sensor Network RAWSN, IEEE, 2015, pp. $42-47$.

[28] S. Chouhan, R. Bose, M. Balakrishnan, Integrated energy analysis of error correcting codes and modulation for energy efficient wireless sensor nodes, IEEE Trans. Wirel. Commun. 8 (2009) 5348-5355.

[29] T. Richardson, R. Urbanke, Efficient encoding of low-density parity-check codes, IEEE Trans. Inf. Theory 47 (2001) 638-656.

[30] D.M. Pham, S.M. Aziz, On efficient design of LDPC decoders for wireless sensor networks, J.Netw. 9 (2014) 3207-3214.

[31] S.M. Choi, B.H. Moon, Implementation of energy efficient LDPC code for wireless sensor node, in: Communication and Networking, 266, Springer, Berlin Heidelberg, 2011, pp. 248-257.

[32] O. Erikson, E. Bjornemo, A. Ahlen, M. Gidlund, On hybrid adaptive forward error correction in wireless sensor networks, in: Annual Conference of the IEEE Industrial Electronics Society, 2011, pp. 3004-3010.

[33] N. Javaid, O. Rehman, N. Alrajeh, Z.A. Khan, B. Manzoor, S. Ahmed, Aid: an energy efficient decoding scheme for LDPCcodes in wireless body area sensor networks, in: Proceedings of International Workshop on Communications and Sensor Networks, 2013, pp. 449-554.

[34] D. Schmidt, M. Berning, N. Wehn, Error correction in single-hop wireless sensor networks a case study, in: Proceedings of Design, Automation, and Test in Europe Conference and Exhibition, 2009, pp. 1296-1301.

[35] L. Zhong, J. Rabaey, A. Wolisz, Does proper coding make single hop wireless sensor networks reality: the power consumption perspective, in: Proceedings of IEEE Wireless Communications and Networking Conference, IEEE, 2005, pp. 664-669.
[36] B. Sarvi, H.R. Rabiee, K. Mizanian, An adaptive cross-layer error control protocol for wireless multimedia sensor networks, Ad Hoc Netw. 56 (2016) 173-185

[37] N. Abughalieh, K. Steenhaut, A Nowe, A Anpalagan, Turbo codes for multi-hop wireless sensor networks with decode-and-forward mechanism, EURASIP J. Wirel. Commun. Netw. 2014 (2014) 1-13.

[38] I. Ez-zazi, M. Arioua, A.E. Oualkadi, P. Lorenz, A hybrid adaptive coding and decoding scheme for multi-hop wireless sensor network, Wirel. Pers. Commun. 94 (4) (2016) 3017-3033.

[39] M. Arioua, Y. Assari, I. Ez-zazi, A.E. Oualkadi, Multi-hop cluster based routing approach for wireless sensor networks, in: International Conference on Ambient Systems, Networks and Technologies, Madrid, 2016, pp. 584-591.

[40] J.C. Giacomin, L.H.A. Correia, T. Heimfarth, G.M. Pereira, V.F. Silva, J.L.P.D. Santana, Radio channel model of wireless sensor networks operating in $2.4 \mathrm{ghz}$ ism band, INFOCOMP J. Comput. Sci. 9 (2010).

[41] T. Rappaport, Wireless Communications: Principles and Practice, Prentice-Hall, Inc., New Jersey, 1996.

[42] W.B. Heinzelman, Application-Specific Protocol Architectures for Wireless networks, Ph.D. thesis. Massachusetts Institute of technology, 2000.

[43] W.B. Heinzelman, A.P. Chandrakasan, H. Balakrishnan, An application specific protocol architecture for wireless microsensor networks, IEEE Trans. Wirel. Commun. 1 (2002) 660-670.

[44] S. Chouhan, R. Bose, M. Balakrishnan, A framework for energy consumption-based design space exploration for wireless sensor nodes, IEEE Trans Comput. Aided Des.Integr. Circ. Syst. 28 (2009) 1017-1024.

[45] S. Cui, A.J. Goldsmith, A. Bahai, Energy-constrained modulation optimization, IEEE Trans. Wirel. Commun. 4 (5) (2005) 2349-2360.

[46] V. Nithya, B. Ramachandran, V. Bhaskar, Energy efficient coded communication for IEEE802.15.4 compliant wireless sensor networks, Wirel. Pers. Commun. 77 (2014) 675-690.

[47] Z.I. Kiss, Z.A. Polgar, M.P. Stef, V. Bota, Improving transmission reliability in wireless sensor networks using network coding, Telecommun. Syst. 59 (2014) 509-521.

[48] R.G. Gallager, Low-density parity-check codes, IRE Trans. Inf. Theory 8 (1962) 21-28.

[49] R.M. Tanner, A recursive approach to low complexity codes, IEEE Trans. Inf. Theory 27 (1981) 533-548.

[50] D.J.C. MacKay, Good error-correcting codes based on very sparse matrices, IEEE Trans. Inf. Theory 45 (2002) 399-431.

[51] Y. Sankarasubramaniam, I.F. Akyuildiz, S.W. McLaughin, Energy efficiency based packet size optimization in wireless sensor networks, in: Proceedings of IEEE International Workshop on Sensor Network Protocols and Applications SNPA'03, IEEE, 2002, pp. 1-8.

[52] S.-M. Choi, B.-H. Moon, Performance analysis on wireless sensor network using LDPC codes over node-to-node interference, in: Proceedings of the International Technical Conference on Circuits Systems, Computers and Communications (ITC-CSCC), 2006, pp. 461-464. 


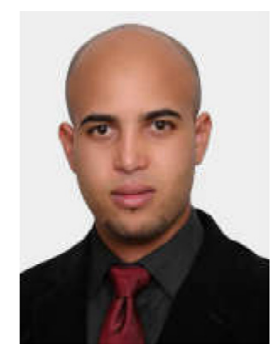

Imad Ez-zazi received the M.Sc. in Telecommunications engineering from National School of Applied Sciences, Abdelmalek Essaadi University in 2014. He is currently a Ph.D. student at the Department of Information and Communication Systems, National School of Applied Sciences (Tangier). His research interests include signal processing, in-network processing techniques for WSNs, design of energy efficient protocols for WSNs and IoT devices, channel and network coding in wireless communications.

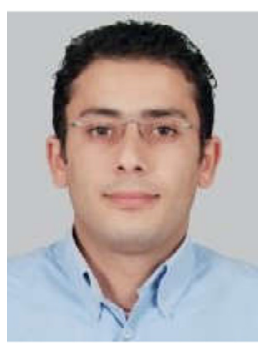

Mounir Arioua received his Ph.D. degree in Telecommunications and Computer Science from Cadi Ayyad University of Marrakech in 2012. In 2012, he joined the National School of Applied Sciences of Marrakech as research assistant and assistant professor. Currently, he is professor at National School of Applied Sciences in the department of Engineering Technologies, University of Abdelmalek Essaadi. His research interests include wireless sensor networks, Internet of things, wireless networking and communication, wireless communications and mobile computing, real-time processing and embedded systems.



Ahmed El Oualkadi received Ph.D. degree from Poitiers University, France, in 2004. From 2000 to 2003, he was a research assistant at the National Higher Engineering School of Poitiers. In 2004, he was an assistant professor at University Institute of Technology, Angoulme. In 2005, he joined the Catholic University of Louvain, Belgium. Currently, he is an associate professor at national school of applied sciences of Tangier, Abdelmalek Essaadi University. His main research interest is RFIC design for wireless communication, wireless sensor networks, embedded system applications and information technology.



Pascal Lorenz received a Ph.D. degree from the University of Nancy, France. Between 1990 and 1995 he was a research engineer at WorldFIP Europe and at Alcatel-Alsthom. He is a professor at the University of Haute-Alsace and responsible of the Network and Telecommunication Research Group. His research interests include wireless sensor networks, QoS, wireless networks and high-speed networks. He is the author/co-author of 3 books, 3 patents and 200 international publications in refereed journals and conferences. 